\title{
Universality of the Fluorescence Intermittency in Nanoscale Systems: Experiment and Theory
}

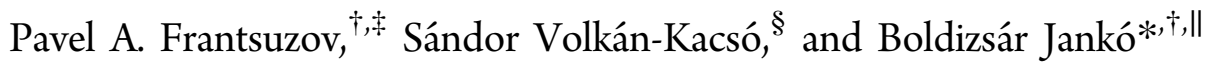 \\ ${ }^{\dagger}$ Department of Physics, University of Notre Dame, Notre Dame, Indiana 46556, United States \\ ${ }^{\ddagger}$ Novosibirsk State University, 630090 Novosibirsk, Russia \\ ${ }^{\S}$ Noyes Laboratory, Chemistry Department, California Institute of Technology, Pasadena, California 91125, United States \\ "Institute for Theoretical Sciences, University of Notre Dame, Notre Dame, Indiana 46556, United States
}

\section{Supporting Information}

\begin{abstract}
A variety of optically active nanoscale objects show extremely long correlations in the fluctuations of fluorescence intensity (blinking). Here we performed a systematic study to quantitatively estimate the power spectral density (PSD) of the fluorescence trajectories of colloidal and self-assembled quantum dots (QDs), nanorods (NRs), nanowires (NWs), and organic molecules. We report for the first time a statistically correct method of PSD estimation suitable for these systems. Our method includes a detailed analysis of the confidence intervals. The striking similarity in the spectra of these nanoscale systems, including even a "nonblinking" quantum dot investigated by Wang and collaborators (Nature 2009, 459, 685-689), is powerful evidence

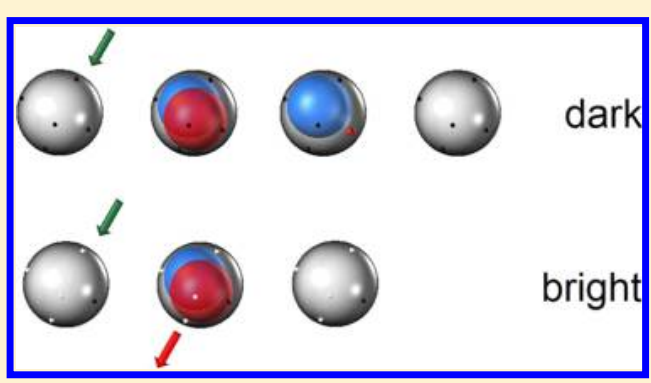
for the existence of a universal physical mechanism underlying the blinking phenomenon in all of these fluorophores (Frantsuzov et al. Nat. Phys. 2008, 4, 519-522). In this paper we show that the features of this universal mechanism can be captured phenomenologically by the multiple recombination center model (MRC) we suggested recently for explaining single colloidal QD intermittency. Within the framework of the MRCs we qualitatively explain all of the important features of fluorescence intensity fluctuations for a broad spectrum of nanoscale emitters.
\end{abstract}

KEYWORDS: Blinking, quantum dot, nanorod, nanowire, organic dye, recombination centers

Since the discovery and development of single molecule $\checkmark$ spectroscopy, ${ }^{3}$ nanoscale imaging underwent rapid advances. A wide variety of optically active nanoscale systems has been studied extensively during the past decades. The resulting massive amount of experimental data imposes severe constraints on a theoretical model aimed at explaining the fluorescence fluctuations of optically active nanoscale systems. In contrast to the detailed experimental characterization, there are very few models that can capture the key features of the experimental data. In particular, fluctuations of the fluorescence intensity on long time scales, from milliseconds to hours, still remain unexplained.

Let us first look at single colloidal quantum dots (QDs) $)^{2,4,5}$ and compare this case later with the other fluorescent nanoscale systems. Fluorescence intensity fluctuations in colloidal QDs were initially explained by the so-called charging mechanism, ${ }^{6}$ connecting the "on" (bright) and "off" (dark) durations to the neutral and the ionized QD states, respectively. However, by now there is sufficient experimental evidence indicating that the charging mechanism cannot explain several features of the QD blinking phenomenon. ${ }^{7-11}$ These recent experimental findings inspired several alternative theoretical explanations which attribute the fluctuations of the emission intensity to variations of the nonradiative relaxation rate of the excited state induced by atomic rearrangements in the $\mathrm{QD}$ surface layer. ${ }^{12-15}$
The multiple recombination center (MRC) model we proposed recently in conjunction with $\mathrm{QD}$ blinking ${ }^{14,15}$ suggests the following mechanism of nonradiative relaxation (Figure 1). The excited state in a QD relaxes by hole trapping to one of $N$ recombination centers (RCs) followed by slower (approximately microseconds) nonradiative recombination of the trapped hole with the remaining electron. The RCs may correspond to the structural defects on the QD surface or at the core/shell interface. Each RC has two conformations, an active and inactive one, corresponding to a high and a low trapping rate, respectively. The total nonradiative recombination rate $k_{\mathrm{t}}$ is given by the sum of individual fluctuating $\mathrm{RC}$ trapping rates $k_{n}$

$$
k_{\mathrm{t}}(t)=\sum_{n=1}^{N} k_{n} \sigma_{n}(t)+k_{0}
$$

Here $\sigma_{n}$ equals 1 when the $n^{\text {th }} \mathrm{RC}$ is in the active conformation and 0 otherwise, while $k_{0}$ is the background rate, that is, when all RCs are in their passive state. Each RC can be described as a stochastic two-level system and performs telegraph noise-like

Received: September 25, 2012

Revised: December 17, 2012

Published: December 31, 2012 


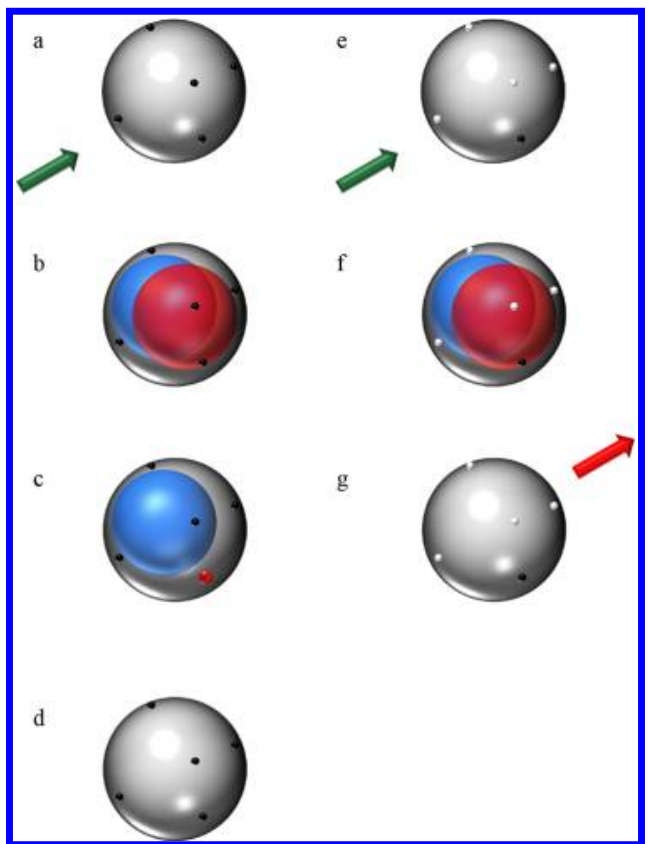

Figure 1. Mechanism of QD fluorescence fluctuations (blinking) within the MRC model. Panels a-d describe the dark QD state, whereas panels $\mathrm{e}-\mathrm{f}$ correspond to the bright state of the QD. (a) The QD (gray sphere) is in the ground electronic state and absorbs a photon (green arrow). (b) An electron-hole pair is generated (blue/ red spheres correspond to the delocalized electron/hole wave functions, respectively). (c) The hole is trapped in one of the active recombination centers (black dot). (d) The electron nonradiatively recombines with the trapped hole. (e) Excitation of the QD ground state after a macroscopic time interval, when most of the recombination centers are switched to the inactive conformation (white dots). (f) Hole trapping is not efficient in the excited state. (g) Recombination to the ground state accompanied by photon emission.

transitions between the active and passive conformation, with rates $\gamma_{n}^{-}$(active to passive) and $\gamma_{n}^{+}$(passive to active). These switching rates are widely distributed, and their distribution controls the power spectral density (PSD) of the blinking trajectory. Following the conventional theory of the multiscale noise in a large class of charge conducting materials $(1 / f$ noise), ${ }^{16}$ we assume that the switching rates have random values and that their distribution is generated by a homogeneous distribution of the energy barrier heights separating the two states of the individual RCs. In the original MRC model we simulated this distribution by a set of rates forming a geometric series. ${ }^{14}$ The generalized MRC framework allows for a slightly different distribution of the "main series" of the RC switching rates as well as for an additional "special" RC with peculiar $\gamma_{i}$ and $k_{i}$ rates. The times spent by the $n^{\text {th }} \mathrm{RC}$ in either conformation are much longer than the time scales describing electronic transitions. We consider low enough excitation intensity such that the lifetime of the trapped species is shorter than the average time between excitations. Under these conditions a steady-state expression applies to the fluorescence intensity ${ }^{12}$

$$
I(t) \sim \frac{1}{1+k_{\mathrm{t}}(t) / k_{\mathrm{r}}}
$$

where $k_{\mathrm{r}}$ is the radiative recombination rate of the excited state. Consequently, it is the fluctuation of the nonradiative rate from eq 1 that in turn results in the fluctuation of emission intensity in eq $2 .{ }^{12}$ In our model, blinking is considered to be precisely this fluctuation over a continuous range of intensities rather than a simple telegraph-like jump between two (an "on" and an "off") states.

If we identify the initial state with equilibrium, it is possible to calculate equilibrium expectation values of quantities such as "on" and "off" distribution, ${ }^{14}$ correlations between subsequent "on" and "off" events, ${ }^{15}$ intensity autocorrelation functions and PSDs, and in principle any higher order version of these quantities (for additional details see the Supporting Information, SI). The MRC model, as opposed to other models of blinking, can explain important experimental observations, such as (1) the continuous distribution of emission intensities and excitation lifetimes, (2) the strong threshold dependence of the "on" and "off" power-law exponents, (3) the long-range correlations between consequent blinking times, and (4) the fact that the fluorescence intensity distribution can have one peak, two peaks, or, as shown below, three peaks in certain QD systems.

Let us now use the concepts and the blinking mechanism introduced within the original MRC model to describe fluorescence intensity not only in colloidal QDs but also in other fluorescent nanoscale systems. The fluorescence intensity trajectories of seven different kind of single fluorophores is plotted in Figure 2, left ("a") panels with corresponding intensity histograms in the central ("b") panels. All of these trajectories can be qualitatively explained within the MRC framework by relaxing some of the constraints introduced in the original model, as mentioned above. We use the term "MRC framework" for the resulting generalized theory to distinguish from the "original" or "basic" MRC model. The emission intensity fluctuations in each case can be successfully modeled with only a handful of interacting RCs (as defined in ref 14 and also given in the SI). The right panels in Figure 2 show the PSD of each trajectory estimated by a unified, nonparametric approach, independent of our model. The experimental PSDs were superimposed with theoretically calculated spectra from the MRC framework (Figure 2, column c) with parameters assuming values in compliance with the basic physical properties of the blinking phenomenon.

The PSD, a quantity extensively used in analyzing stochastic time series, was first introduced into the field of single particle fluorescence by Pelton et al. in the context of QD blinking. ${ }^{17}$ We believe that this powerful method is superior to others in several respects. Notably, it does not require defining a moreor-less arbitrary intensity threshold, as does the traditional "on/ off" analysis, nor does it show any dependence at relevant time scales on the binning time in stark contrast with the intensity histogram. ${ }^{18-20}$

On one hand, to calculate the theoretical PSD, we calculate the time autocorrelation function and then apply the WienerKhinchine theorem. The autocorrelation function is calculated from the two-time conditional probability in the configuration space of the $N$ RCs. In this space the time-dependent behavior of the RCs can be described as a Markov process; hence the two-time conditional probability is the propagator itself.

On the other hand, our method of PSD estimation from experimental trajectories relies on the modified averaged periodogram (MAP) estimator. In addition to defining and applying a so-called point estimator for the PSD (circles in Figure 2, panels c) we provided confidence intervals (error bars in Figure 2, panels c). Due to the confidence intervals, we are able to draw robust conclusions such as the $1 / f^{r}$ nature of the 


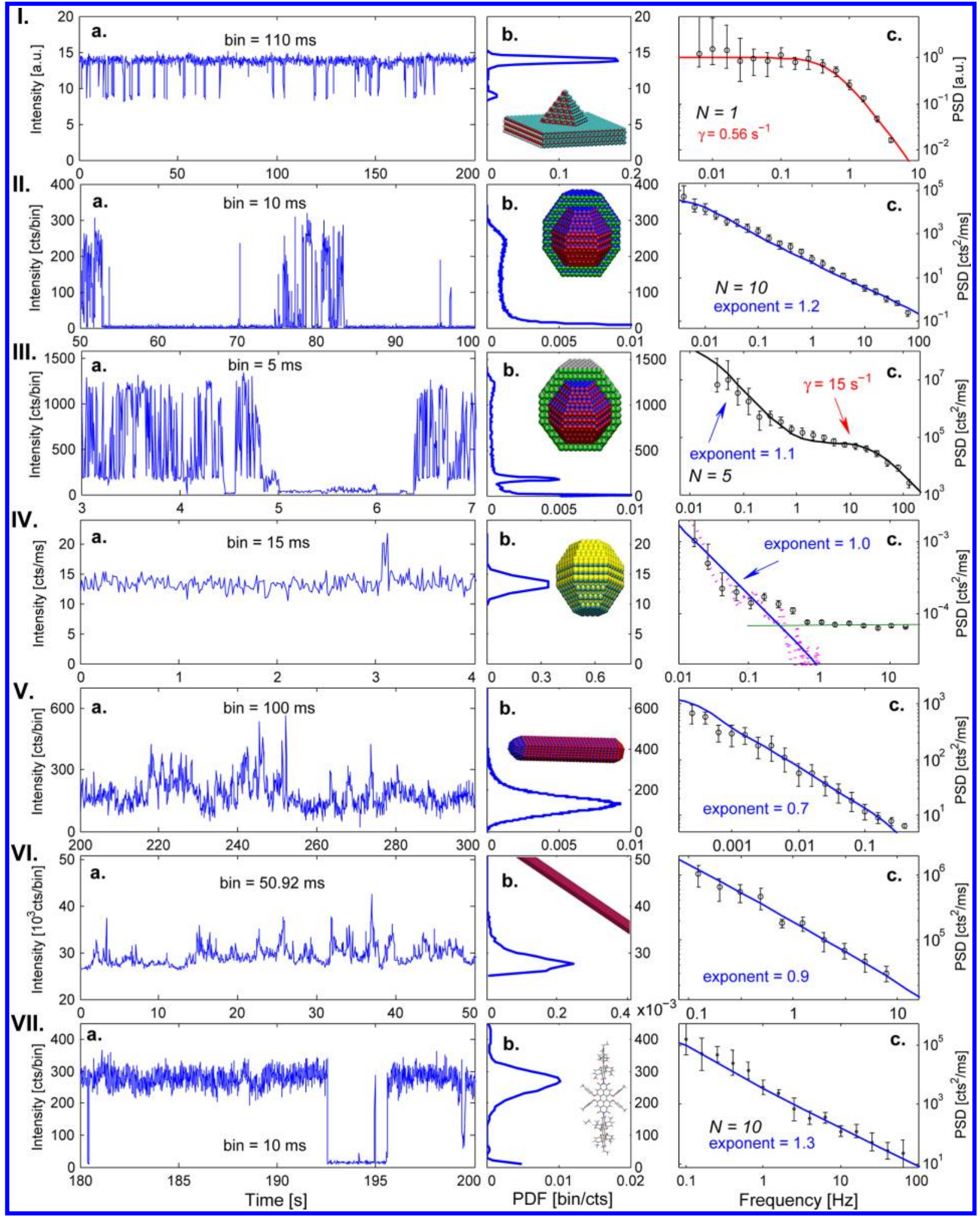

Figure 2. Fluorescence intermittency in various nanoscale fluorophores. Experimental fluorescence trajectories (panels a), with the corresponding intensity histograms (panels b) and power spectral densities (panels c, circles, experiment; solid lines, theory) are shown for (I) a single selfassembled InP QD (ref 22), (II) a colloidal CdSe/ZnS (core/shell) QD (ref 14), (III) a colloidal CdSe/CdS (core/shell) QD (ref 28), (IV) a CdZnSe/ZnSe "nonblinking" QD (ref 1), (V) a colloidal CdSe/ZnSe NR (ref 29), (VI) a CdSe NW (ref 30), and (VII) a perylene diimide molecule (ref 31). For all panels c, solid blue lines show $1 / f^{r}$-type PSDs calculated from the MRC framework, whereas the black line in panel III.c was calculated with specially chosen RCs (we refer the reader to the SI for details). The red line in panel I.c is a Lorentzian fit to the PSD corresponding to a single RC, while the green line and the dotted magenta line on the panel IV.c show shot noise and signal spectra after filtering, respectively. The insets in panels b show the schematic structure of each fluorescent nanoscale system. The PSD in panels $\mathrm{c}$ were obtained from a modified average periodogram method. The error bars correspond to a 95\% confidence interval. Further details on the statistical analysis can be found in the SI. 
analyzed spectra. For consistency we apply a single unified method to all trajectories, even though the fluorescence fluctuations show significant variation between systems. The method developed and applied here is nonparametric and thus model-free. This means that it is independent of-and unbiased by-the MRC model.

The elements of the spectral estimation are well-known in the literature and can be applied in a straightforward manner to analyze each trajectory. Nevertheless, the standard MAP method had to be tuned and further refined (1) to address the presence of many time scales, (2) to yield unbiased results, and (3) to provide reasonable confidence intervals. In the MAP method the first step is to apply a suitably defined time window function to the original time series (trajectory), an operation which is equivalent to partitioning the trajectory in smaller pieces. Next, for any chosen frequency the periodogram (a Fourier estimation of the PSD) is calculated for each of these segments. Note that these segments are allowed to overlap. Finally, the periodograms are averaged.

We have found a criterion to chose an optimal window size and overlap that allows for an unbiased estimation of the PSD. The optimal window size greatly varies depending on the frequency at which the PSD is to be estimated. In estimating the confidence intervals we have taken into account that the individual periodograms are not statistically independent. In finding the probability distribution of the point estimator we relied on a generalized version of the central limit theorem. A detailed description of our methods to calculate the theoretical PSD and estimate the experimental PSD is available as part of the SI. Below, we discuss the specific features of each nanoscale fluorophore with a special emphasis on the PSD of their blinking trajectories.

Self-assembled InP QDs (Figure 2, I.a-c) under continuous excitation sometimes yield telegraph noise-like stochastic trajectories characterized by two intensity levels. ${ }^{21,22}$ The fluctuation dynamics can be easily explained by taking the limiting case of the MRC model when there is only one RC ( $N$ $=1)$. This case corresponds to a Lorentzian PSD, in excellent agreement with the experimental data (cf. panel I.c of Figure 2).

Colloidal CdSe/ZnS (core/shell) QDs (Figure 2, II.a-c) exhibit a very wide range of time scales in their fluorescence intermittency, from hundreds of microseconds to thousands of seconds. ${ }^{18}$ The PSD of these blinking trajectories show a distinct power law frequency $(f)$ dependence, ${ }^{17,23}$ namely, $\sim 1 /$ $f^{r}$, where the exponent $r$ is usually slightly greater than unity. The particular trajectory shown in Figure 2, panel II.c yielded $r$ $\approx 1.2$. It is remarkable that the fluorescence intermittency in these QDs can be regarded as the optical analogue of the wellknown $1 / f$ noise. $^{16}$ Moreover, the distributions of the "on" and "off" durations from the traditional threshold method systematically exhibit a power-law dependence with an exponential tail, $^{2,4,5,18,24,25}$

$$
p_{\text {on } / \text { off }}(t) \sim t^{-m} \exp (-t / T)
$$

We found recently ${ }^{14}$ that the threshold analysis, although very useful, is an incomplete tool for the characterization of blinking: the parameters of the "on" and "off" distributions strongly depend on the more-or-less arbitrarily defined threshold. This dependence, the $1 / f$-like power spectrum of the fluorescence intensity fluctuations, ${ }^{17,23}$ and the long-range correlations between consequent blinking times observed by Stefani et al. $^{26}$ was qualitatively explained by the MRC model. ${ }^{14,15}$ For modeling this class of nanoscale blinkers we followed our previous work ${ }^{14}$ and assumed $10 \mathrm{RCs}$ with switching times homogeneously distributed on logarithmic scale. This condition is satisfied if the rates follow the geometric sequence

$$
\gamma_{i}=\gamma_{1} a^{i-1}
$$

where $a$ controls the number of RCs per decade of time. A set of parameters (given in the SI) similar to those in ref 14 yielded here a PSD which is in excellent agreement with the experiment (Figure 2, II.c). This distribution arises most likely from the surface atoms of the QD switching between a variety of local crystal configurations. We propose that the sizes of the tunneling barriers between quasi-stable positions follow a homogeneous distribution which is consistent with eq 4.

Colloidal CdSe/CdS (core/shell) QDs (Figure 2, III.a-c) have suppressed blinking behavior with well-defined high, medium, and low ("off") intensity levels. ${ }^{27,28}$ The PSD has a Lorentzian high-frequency asymptotic behavior corresponding to fast transitions between high and medium levels and a $1 / f^{r}(r$ $=1.1$ ) dependence at low frequencies. Within the MRC framework this corresponds to one "weak" RC with low trapping rate and a few "strong" RCs with high trapping rates in the active conformation. The transitions between the active and inactive conformations of the "weak" RCs generate emission intensity jumps between the high and medium levels. The activation of any "strong" RC induces low fluorescence level. In Figure 2, III. c, we attained an excellent agreement with the experimental PSD by assuming a relatively fast switching rate of $\gamma=15 s^{-1}$ for the "weak" RC, and a typically distributed set of four "strong" but slow RCs following eq 4 . We refer the reader to the SI for more details on the fitting model parameters. The relatively small number of RCs needed to explain blinking in $\mathrm{CdSe} / \mathrm{CdS}$ QDs may be due to the fact that the lattice mismatch in this structure is smaller than in the $\mathrm{CdSe} / \mathrm{ZnS}$ core/shell QDs.

Alloyed $\mathrm{CdZnSe} / \mathrm{ZnSe}$ QDs (Figure 2, IV.a-c) show no "off" events ${ }^{1}$ and provide the ultimate example of "nonblinking" dots. ${ }^{32}$ Originally, it was proposed that the intensity fluctuations of this particular QD are caused by shot noise only. However, our PSD estimation in Figure 2, IV.c does not support this assumption. The spectral density has a $1 / f$ dependence, gradually suppressed by white noise at high frequencies. After filtering out the white noise (Figure 2, IV.c, dotted curve), it becomes obvious that this power law dependence extends to more than two decades in time. Note that a recent paper demonstrated power law blinking statistics in $\mathrm{CdSeS} / \mathrm{ZnS}$ alloyed-core/shell type structures ${ }^{33}$ which is consistent with the $1 / f$ type PSD. ${ }^{14}$ The reduced blinking of the alloyed QDs are easily explained even within the original MRC model, provided that we choose small RC trapping rates relative to the radiative relaxation rate. We speculate that the suppressed trapping rates may be related to the way in which these alloyed QDs are synthesized. Indeed, the procedure includes annealing, which in turn may move the defects to the surface, far from the hole confined in the center, and could lead to weaker trapping rates. The high contribution of the shot noise prevents us from providing an accurate number of RCs. A theoretical PSD calculated from only seven RCs is, nevertheless, in good agreement with the experiment.

$\mathrm{CdSe} / \mathrm{ZnSe}$ NRs (Figure 2, V.a-c) have no well-defined "on" fluorescence intensity level. ${ }^{20,29}$ The NR intensity trajectories show spikes, a feature not found in typical CdSe/ 
ZnSe QD trajectories (cf. Figure 2, II.a). Nevertheless, the MRC framework can explain NR blinking as well. The distribution of "on" and "off" durations have the truncated power-law form given in eq $3 .^{20,29}$ Remarkably, the parameters $m$ and $T$ show a threshold dependence ${ }^{20}$ that is very similar to the one we discovered in CdSe/ZnS QDs. The absence of the "on" emission level in NRs is likely due to a larger number of surface defects located on a relatively large surface area. Furthermore, the PSD has, once again, the typical $1 / f^{r}$ form (Figure 2, V.c). $N=12$ RCs can fit this PSD well, although their true number is probably larger. The exponent $r=0.7$ is significantly lower than 1 . After thoroughly exploring the parameter space of the MRC model, as formulated originally in ref 14, we empirically concluded that the switching rates described by eq 4 is not consistent with the $r<1$ PSD. Therefore, we chose switching rates that were power-law distributed following a $\gamma^{-r}$ dependence. For more details on the $\gamma^{-r}$ distribution, see the SI.

CdSe NWs (Figure 2, VI.a-c) exhibit no clear "off" state and therefore are similar to alloyed QDs. The spatially inhomogeneous ${ }^{34}$ fluorescence seen in a typical wire is an intriguing phenomenon specific to the NW fluorescence and will be discussed elsewhere. However, some NWs, called "whole blinkers", show correlated fluorescence fluctuations along their full length. ${ }^{30}$ The analyzed wire is characterized by a $1 /$ $f^{r}(r=0.9)$ fluctuation spectrum and shows similarity to NRs in the sense that the exponent is smaller than 1 (Figure 2, VI.c). Therefore, this system was modeled assuming a $\gamma^{-r}$ switching rate distribution within a noninteracting model of $10 \mathrm{RCs}$. We believe that the spatially resolved intensity fluctuations along NWs other then whole blinkers ${ }^{34}$ can also be explained within the MRC framework, provided that we account for the possibility of multiple carrier excitation.

Perylene diimide molecules (Figure 2, VII.a-c) embedded into a glassy environment have emission properties that are very similar to those of the colloidal CdSe/ZnSe QDs. ${ }^{31}$ These molecules have well-defined "on" and "off" intensity levels and a $1 / f^{r}(r=1.3)$ PSD. Within the MRC framework, these fluorescence fluctuations can be modeled with $N \sim 10$ RCs. The blinking of these molecules cannot be viewed as a version of the random telegraph noise with a well-defined "on" and "off" state, rather it is a consequence of the fluctuating nonradiative rate. We speculate that the RCs in this case are mobile atomic defects of the host material located in the close proximity of the molecule.

Above we have shown how each of the seven trajectories can be explained within the MRC framework in a unified manner. Remarkably, the $1 / f^{r}$-type (power-law) PSD is a common feature of the analyzed trajectories with the exception of the self-assembled QD (Figure 2, I). The exponent $r$ varies from fluorophore to fluorophore and assumes values ranging from 0.7 (NR) to 1.3 (dye trimer molecule). We have shown that the $1 / f^{r}$-type spectra of the "small" fluorophores [QDs (Figure 2, II-IV) and the perylene diimide molecule (Figure 2, VII)] all yield $r \geq 1$. These PSDs can be easily explained by the interacting MRC model in which the logarithm of the switching rates are uniformly distributed according to eq 4 .

However, the "large" fluorophores [the NR and NW (Figure 2, V-VI)] having exponents smaller than 1 required a slight relaxation in the structure of the "basic" MRC model originally formulated for $\mathrm{CdSe} / \mathrm{ZnS} \mathrm{QDs}$ by allowing for a $\sim \gamma^{-r}$ distribution of RC switching rates. Note, however that both the NR and the NW trajectories resulted from measurements using charge-coupled device (CCD) cameras. ${ }^{29,30}$ Meanwhile all other nanoblinkeres were investigated by single photon counting techniques. We speculate that the reduced power law exponent of the NR and NW PSDs may have resulted from measurement-related artifacts. For example, the NR trajectory from Figure 2, V.a has been preprocessed by Drndic and coworkers, who subtracted from the trajectories a fourth order polynomial fit to the background. ${ }^{29}$ This preprocessing was deemed necessary to reduce the strong background noise level. It is equivalent to an effective removal of low frequency components in the PSD probably leading to a decreased power law exponent. Note also that the power law exponent of the NW is only marginally smaller than 1 . Consequently, we cannot rule out the possibility that the unbiased exponents for both the NR and NW are in fact $\sim 1$, in which case the distribution from eq 4 could explain the NR and NW trajectories as well.

The seven nanoscale systems analyzed in this paper were all probed by continuous wave excitation at relatively low excitation intensities. Such experimental conditions most likely resulted in a negligible probability associated with multiexciton states. Furthermore, the blinkers we studied here were not connected to any source of charge. However, there are cases, such as in experiments performed in an electrochemical cell, $^{8,33,35}$ or in combined optical and electrostatic force microscopy measurements, ${ }^{36}$ where charge exchange between the blinker and its environment plays an important role. More recently, these and other single fluorophore systems were subjected to pulsed excitation using time tagged time-resolved spectroscopy techniques. These techniques allow for a greater control over the excitation conditions (pulse intensity and rate) and reveal the time dependence of the relaxation processes at a single particle level. The findings reported in these experiments are consistent with the MRC framework.

Recent investigation of the relaxation of excitons in the "off" state revealed that this process depends on the excitation intensity even below saturation and that the relaxation of charge-separated species is much faster then the long time scales of blinking. ${ }^{10}$ This is consistent with the assumptions we made and the results we obtained previously within our MRC model. In particular, we assumed that blinking is light-induced and results from the fluctuation of the nonradiative rate, rather than charging-discharging events. Moreover, the instantaneous "off" lifetimes (lifetime blinking) were shown to be correlated in a linear fashion with the corresponding intensity, ${ }^{11}$ as predicted by the MRC model. ${ }^{37}$ Systems with reduced blinking such as "giant" CdSe/CdS QDs also follow power law kinetics. ${ }^{38}$ Again, our model is consistent with these findings. Indeed, placing the surface RCs farther from the hole localized inside the core would result in a reduced blinking as trapping rates become much smaller than the radiative relaxation rate. Similarly, opening an efficient channel for energy transfer by manipulating the QD environment also results in a reduction of blinking and concomitantly of quantum yield, as observed in the experiment. ${ }^{39,40}$

Finally, we mention four new experimental studies that are consistent with our model. One study reports on how the blinking behavior of single QDs depends on both pulse intensity and repetition rate, revealing a number of multiexciton effects at large laser power. ${ }^{41}$ In the other two studies probing of charge separated (trion) states in conjunction with blinking was made possible by pulsed excitation and electrochemical techniques elegantly combined with single QD spectroscopy. ${ }^{33,35,42}$ Note that systems studied in these experiments are 
known to follow the typical power-law statistics, indicative of compatibility with the MRC framework. More theoretical investigation is required to understand the properties of states involving trions as well as multiexcitonic states resulting from a slow relaxation of trapped holes and an increased pulse excitation intensity.

In conclusion we presented a phenomenological theoretical framework that captures for the first time all of the essential features of a wide class of fluorescent nanoscale systems. This framework is based on our MRC model originally proposed for colloidal $\mathrm{CdSe} / \mathrm{ZnS}$ QDs, and it succeeds in describing semiquantitatively the fluorescence emission in nanoscale objects like QDs, NRs, NWs, and organic molecules in a glassy environment. Despite clear differences in their structure, dimensionality, and environment, our paper demonstrates that all the fluorophores we looked at exhibit important common features. The fact that these features of nanoscale fluorescence are naturally explained by our framework suggests that the microscopic mechanism behind the intensity fluctuations in each individual class of nanoscale fluorophores will also be similar. Thus, our phenomenological theoretical framework provides an important step toward a microscopic model of nanoscale fluorescence. The result presented in this paper suggests that substantial progress can be made by aiming experimental and theoretical efforts directly at revealing the microscopic nature and properties of RCs in each class of nanoscale fluorophores.

\section{ASSOCIATED CONTENT}

\section{S Supporting Information}

Further details on the MRC formalism, the calculation of the theoretical PSD, and the estimation of the theoretical power law exponents and switching rates (where applicable). The PSD estimation method from experimental trajectories is also presented in detail. This material is available free of charge via the Internet at http://pubs.acs.org.

\section{AUTHOR INFORMATION}

\section{Corresponding Author}

*E-mail: bjanko@nd.edu.

\section{Notes}

The authors declare no competing financial interest.

\section{ACKNOWLEDGMENTS}

The authors would like to thank Drs. C. Crouch, M. Drndic, D.Gomez, J. Hoogenboom, T. Krauss, M. Kuno, R. Loomis, P. Mulvaney, M. Pelton, V. Protasenko, and M. Sugisaki for kindly sharing their experimental data with us. We would also like to acknowledge the support of the Institute for Theoretical Sciences, the Department of Energy, Basic Energy Sciences, and the National Science Foundation via the NSF-NIRT Grant No. ECS-0609249.

\section{REFERENCES}

(1) Wang, X.; Ren, X.; Kahen, K.; Hahn, M. A.; Rajeswaran, M.; Maccagnano-Zacher, S.; Silcox, J.; Cragg, G. E.; Efros, A.; Krauss, T. Nature 2009, 459, 686-689.

(2) Frantsuzov, P. A.; Kuno, M.; Jankó, B.; Marcus, R. A. Nat. Phys. 2008, 4, 519-522.

(3) Moerner, W. E.; Orrit, M. Science 1999, 283, 1670-1676.

(4) Stefani, F. D.; Hoogenboom, J. P.; Barkai, E. Phys. Today 2009, 34-39.
(5) Cichos, F.; von Borczyskowski, C.; Orrit, M. Curr. Opin. Colloid Interface Sci. 2007, 12, 272-284.

(6) Efros, A. L.; Rosen, M. Phys. Rev. Lett. 1997, 78, 1110-1113.

(7) Zhang, K.; Chang, H.; Fu, A.; Alivisatos, A. P.; Yang, H. Nano Lett. 2006, 6, 843-847.

(8) Jha, P. P.; Guyot-Sionnest, P. ACS Nano 2009, 3, 1011-1015.

(9) Zhao, J.; Nair, G.; Fisher, B. R.; Bawendi, M. G. Phys. Rev. Lett. 2010, 104, 157403.

(10) Rosen, S.; Schwartz, O.; Oron, D. Phys. Rev. Lett. 2010, 104, 157404.

(11) Cordones, A. A.; Bixby, T. J.; Leone, S. R. Nano Lett. 2011, 11, 3366-3369.

(12) Frantsuzov, P. A.; Marcus, R. A. Phys. Rev. B 2005, 72, 155321.

(13) Park, S. J.; Link, S.; Miller, W. L.; Gesquiere, A.; Barbara, P. F. Chem. Phys. 2007, 341, 169-174.

(14) Frantsuzov, P. A.; Volkán-Kacsó, S.; Jankó, B. Phys. Rev. Lett. 2009, 103, 207402.

(15) Volkán-Kacsó, S.; Frantsuzov, P. A.; Jankó, B. Nano Lett. 2010, 10, 2761-2765.

(16) Weissman, M. Rev. Mod. Phys. 1988, 60, 537-571.

(17) Pelton, M.; Grier, D. G.; Guyot-Sionnest, P. Appl. Phys. Lett. 2004, 85, 819-821.

(18) Kuno, M.; Fromm, D. P.; Hammann, H. F.; Gallagher, A.; Nesbitt, D. J. J. Chem. Phys. 2001, 115, 1028-1040.

(19) Gopich, I. V.; Szabó, A. J. Chem. Phys. 2006, 124, 154712.

(20) Crouch, C. H.; Sauter, O.; Wu, X.; Purcell, R.; Querner, C.; Drndic, M.; Pelton, M. Nano Lett. 2010, 1692-1698.

(21) Pistol, M.-E.; Castrillo, P.; Hessman, D.; Prieto, J. A.; Samuelson, L. Phys. Rev. B 1999, 59, 10725-10729.

(22) Sugisaki, M.; Ren, H.; Nishi, K.; Masumoto, Y. Phys. Rev. Lett. 2001, 86, 4883-4886.

(23) Pelton, M.; Smith, G.; Sherer, N. F.; Marcus, R. A. Proc. Natl. Acad. Sci. 2007, 104, 14249-142254.

(24) Peterson, J. J.; Nesbitt, D. J. Nano Lett. 2009, 9, 338-345.

(25) Shimizu, K. T.; Neuhauser, R. G.; Leatherdale, C. A.; Empedocles, S. A.; Woo, W. K.; Bawendi, M. G. Phys. Rev. B 2001, 63, 205316

(26) Stefani, F. D.; Zhong, X.; Knoll, W.; Han, M.; Kreiter, M. New J. Phys. 2005, 7, 197.

(27) Mahler, B.; Spinicelli, P.; Buil, S.; Quelin, X.; Hermier, J.-P.; Dubertret, B. Nat. Mater. 2008, 7, 659-664.

(28) Gomez, D. E.; van Embden, J.; Mulvaney, P.; Fernee, M. J.; Rubinsztein-Dunlop, H. ACS Nano 2009, 3, 2281-2287.

(29) Wang, S.; Quemer, C.; Fischbein, M. D.; Willis, L.; Novikov, D. S.; Crouch, C. H.; Drndic, M. Nano Lett. 2008, 8, 4020-4026.

(30) Glennon, J. J.; Tang, R.; Buhro, W. E.; Loomis, R. A. Nano Lett. 2007, 7, 3290-3295.

(31) Hoogenboom, J. P.; Hernando, J.; van Dijk, E. M. H. P.; van Hulst, N. F.; Garsia-Parajo, M. F. Chem. Phys. Chem. 2007, 8, 823833.

(32) Krauss, T. D.; Peterson, J. J. J. Phys. Chem. Lett. 2010, 1, 13771382.

(33) Qin, W.; Shah, R. A.; Guyot-Sionnest, P. ACS Nano 2012, 6, 912-918.

(34) Protasenko, V. V.; Hull, K. L.; Kuno, M. Adv. Mater. 2005, 17, 2942-2949.

(35) Galland, C.; Ghosh, Y.; Steinbruck, A.; Sykora, M.; Hollingsworth, J. A.; Klimov, V. I.; Htoon, H. Nature 2011, 479, 203-208.

(36) Krauss, T. D.; Brus, L. E. Phys. Rev. Lett. 1999, 83, 4840-4843.

(37) Volkán-Kacsó, S. Theory of Fluorescence Intermittency in Quantum Dots and Other Flourophores. Ph.D. Dissertation, University of Notre Dame, Physics Department, Notre Dame, IN, 2011.

(38) Vela, J.; Htoon, H.; Chen, Y.; Park, Y.-S.; Ghosh, Y.; Goodwin, P. M.; Werner, J. H.; Wells, N. P.; Casson, J. L.; Hollingsworth, J. A. J. Biophotonics 2011, 3, 706-717.

(39) Jin, S.; Song, N.; Lian, T. ACS Nano 2010, 4, 1545-1552. 
(40) Chen, Z.; Berciaud, S.; Nuckolls, C.; Heinz, T. F.; Brus, L. E. ACS Nano 2010, 4, 2964-2968.

(41) Schwartz, O.; Tenne, R.; Levitt, J. M.; Deutsch, Z.; Itzhakov, S.; Oron, D. ACS Nano 2012, 6, 8778-8782.

(42) Qin, W.; Guyot-Sionnest, P. ACS Nano 2012, 6, 9125-9132. 